\title{
ABNORMAL OPTICAL PROPERTIES OF BABINGTONITE FROM THE YAKUKI MINE, JAPAN*
}

\author{
TOKUGORO SHODA \\ Mineralogical Department, Faculty of Science, \\ University of Tokyo
}

\begin{abstract}
Abnormal optical properties of babingtonite from the Yakuki mine, Japan, are described. Sections cut nearly normal to each of the optic axes of this mineral show no extinction. This is probably due to elliptic vibration of light which passes through the sections. Dependence of the ellipticity on the orientation of the section was determined. Interference figure exhibited by sections cut nearly normal to one of the optic axes is anomalous, especially remarkable for red light. The mineral absorbs light of different colour in different amount. Dependence of the optical density on the wave lengths of light is shown by variation curves.
\end{abstract}

\section{Introduction}

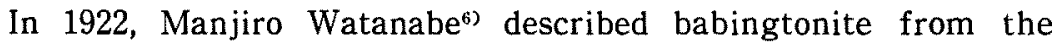
Yakuki mine, Fukushima Prefecture, Japan. He gave detailed morphological data, taking the crystallographic axes after Dauber ${ }^{1)}$. Later, Richmond ${ }^{3)}$ adopted a new axial system of babingtonite according to the normal triclinic setting proposed by Peacock ${ }^{2)}$, and gave the optical orientations of the mineral. Further, he gave the chemical formula of the mineral as $\mathrm{Ca}_{4} \mathrm{Fe}_{2}{ }^{2+} \mathrm{Fe}_{2}{ }^{3+} \mathrm{Si}_{10} \mathrm{O}_{28}(\mathrm{OH})_{2}$ on the basis of its chemical analysis.

Recently, the present writer made some optical examination of

* Read at the joint meeting of the Mineralogical Society of Japan, the Japanese Association of Mineralogists, Petrologists and Economic Geologists, and the Society of Mining Geologists of Japan, held in Kyoto on Oct. 20, 1960. 
babingtonite from the Yakuki mine, and determined its orientations.

In the course of this study, he found that sections cut nearly perpendicular to each of the optic axes (within the range of about $20^{\circ}$ from the optic axis) generally show no extinction, even observed with monochromatic light, and confirm that this phenomenon is due to elliptic vibration of light that passes the mineral in the above-said direction, as the present writer ${ }^{45)}$ has remarked of some alkali amphiboles in his previous papers. In the present paper, the writer intends to give a brief description of the abnormal optical phenomenon of babingtonite.

\section{Morphology and optical orientation}

Morphological and optical data of babingtonite as obtained by the writer and Richmond ${ }^{3)}$, are compared in Table 1. The writer's data are based on his study of specimens, about $1 \sim 3 \mathrm{~mm}$. in size. They are prismatic, with faces belonging to [001]; cleavage: perfect $\{001\}$ and indistinct $\{110\}$.

Table 1. Morphological and optical data of babingtonite.

\begin{tabular}{|c|c|c|c|c|c|}
\hline \multirow{2}{*}{$(h k l)$} & \multicolumn{3}{|c|}{ Yakuki (Shoda) } & \multicolumn{2}{|c|}{ Blueberry Mt. (Richmond) } \\
\hline & $\rho$ & $\varphi$ & $n$ & $\rho$ & $\varphi$ \\
\hline$(010)$ & $90^{\circ} 00^{\prime *}$ & $0^{\circ} 00^{\prime *}$ & 7 & $90^{\circ} 00^{\prime}$ & $0^{\circ} 00^{\prime}$ \\
\hline (110) & 9005 & 4738 & 4 & , & 4736 \\
\hline$(100)$ & 9000 & 7547 & 7 & $"$ & 7549 \\
\hline (11i0) & 8956 & 11204 & 8 & , & 11211 \\
\hline$(1 \overline{2} 0)$ & 8952 & 13621 & 5 & , & 13654 \\
\hline (001) & 425 & 5719 & 4 & 436 & 5639 \\
\hline$+\mathrm{A}$ & $22^{\circ}$ & $-2^{\circ}$ & & $20^{\circ} 55^{\prime}$ & $-5^{\circ} 48^{\prime}$ \\
\hline$+B$ & 86 & 52 & & 8642 & 5320 \\
\hline
\end{tabular}

$n$ : Number of faces measured. * Standard point.

With sections cut nearly normal to each of the optic axes, the orientations of the optic axes with respect to the normals of the 


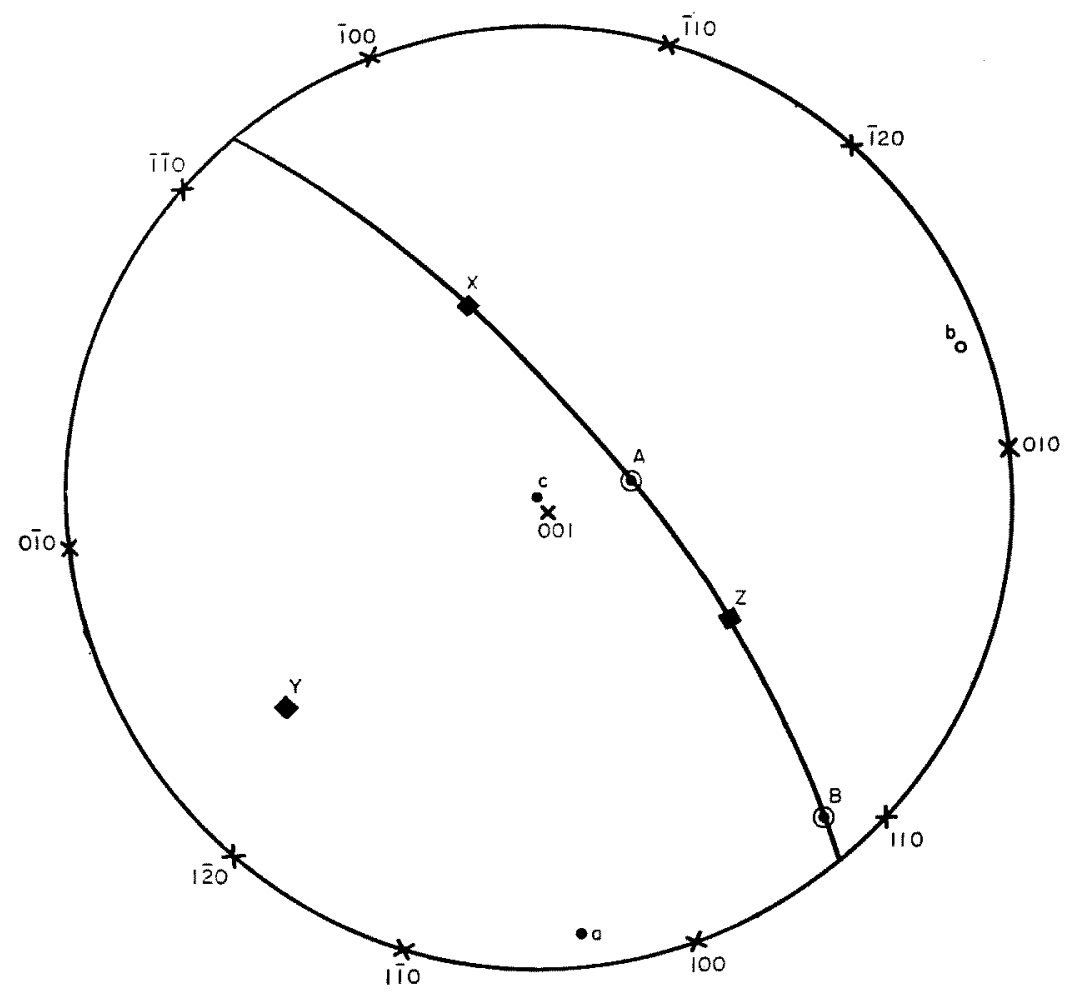

Fig. 1. Stereographic projection showing the optical orientation of Yakuki babingtonite.

sections were determined conoscopically. The results were plotted in stereographic projection as shown in Fig. 1.

\section{Confirmation of elliptic vibration}

A section of an aggregate of babingtonite crystals was examined under the microscope, and a comparatively large grain of the mineral which shows no extinction was selected for optical study. In order to confirm the elliptic vibration of light passing through the grain, it was observed between two $\frac{1}{4} \lambda$ plates, superposed in subtraction position, using the polarizing microscope with three rotation stages, 


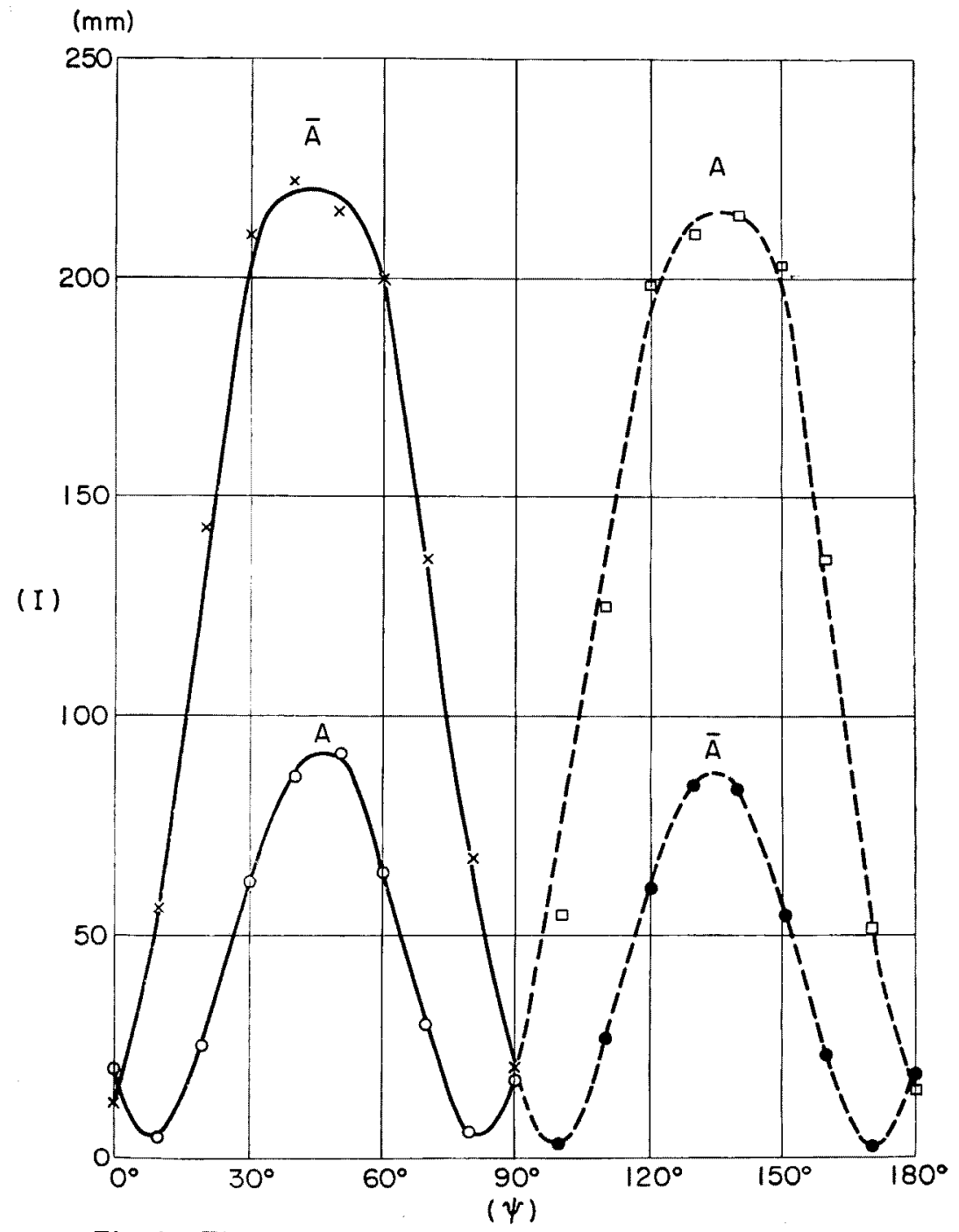

Fig. 2. The values of the minimum intensity for various values of $\Psi$ for the section near the optic axis A (crossed polars).

Full lines: for the right-handed incident polarization.

Broken lines: for the left-handed incident polarization.

$\square$ and $O$ : for sections nearly normal to $+A$.

$X$ and - for section nearly normal to $-A$.

I: Scale readings on galvanometer. 
and the intensity of light transmitted through the grain was measured by means of the photomultiplier-galvanometer-set ${ }^{4}$. Then, the section was turned over and the similar measurement was made for the same grain but in the opposite direction. The result is shown in Fig. 2, where full line represents the curve for right handed incident polarization, and broken line, for the left handed.

From this figure, it is readily seen that the sence of the elliptic vibration is reversed if viewed from the other side of the crystal, while the ellipticity (ratio of the axial lengths of ellipse) remains constant. From the above, it is probable that non-extinction phenomenon of this mineral is related to a centrosymmetrical one and is due to the elliptic vibration of light caused by strong absorption of this mineral. (The orientation and the value of $\psi\left(=+8^{\circ}\right)$ for this grain is shown in Fig. 3.)

\section{Dependence of the ellipticity on orientation}

It is very easy to distinguish optic axis A from B by observing interference figure, of which explanation will be given later. Moreover, it is possible to decide whether the optic axis under observation corresponds to $+\mathrm{A}$ or $-\mathrm{A}$ in direction by determining relative orientations of (1) the optic axis under question, (2) normal of the cleavage plane (\{001\} and/or \{110\}) and (3) bisectrix Y (normal of the optical plane). With twenty-five grains, cut nearly perpendicular to the optic axis, which had been selected under the microscope, accurate determinations were made of their orientations with respect to the optic axis and optical plane, by means of the universal stage. Then, the ellipticity of vibration for each of these grains was determined by the method already described.

Observations were made with grains orientated near the optic axis, in either sense, $+\mathrm{A}$ or $-\mathrm{A}$. The results obtained are shown in Fig. 3 , all reduced to those in $+A$ sence. In Fig. 3 , solid circles, solid squares and solid triangles represent directions with elliptic vibration of positive sign (right handed), the values of $\psi$ (a measure of ellipti- 


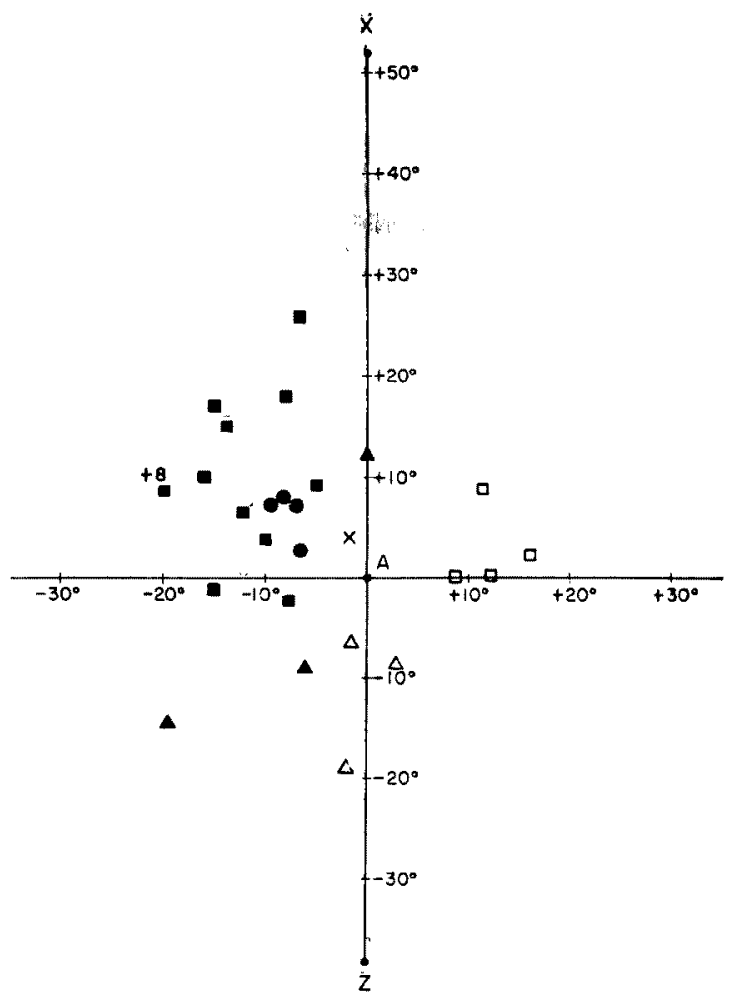

Fig. 3. Dependence of the ellipticity of vibration on orientation near the optic axis $\mathrm{A}$.

Circle: $\quad \Psi_{\min }>10^{\circ} \quad$ Cross: $2^{\circ}>\Psi_{\min }>-2^{\circ}$.

Square: $10^{\circ}>\Psi_{\min }>5^{\circ}$ Solid: Right handed.

Triangle: $5^{\circ}>\Psi_{\min }>2^{\circ}$ Open: Left handed.

city) being respectively $\psi_{\min }>10^{\circ}, 10^{\circ}>\psi_{\min }>5^{\circ}$ and $5^{\circ}>\psi_{\min }>2^{\circ}$. The $\psi$ is the angle between the vibration direction of polarizer and one of the vibration directions of the superposed $\frac{1}{4} \lambda$ plates, and is related to ellipticity $\varepsilon$ as follows;

$$
\varepsilon=\tan \psi \text {. }
$$

Open circles, open squares and open triangles represent directions with elliptic vibration of negative sign (left handed), and crosses represent directions with nearly linear vibration $\left(2^{\circ}>\psi_{\min }>-2^{\circ}\right)$. 


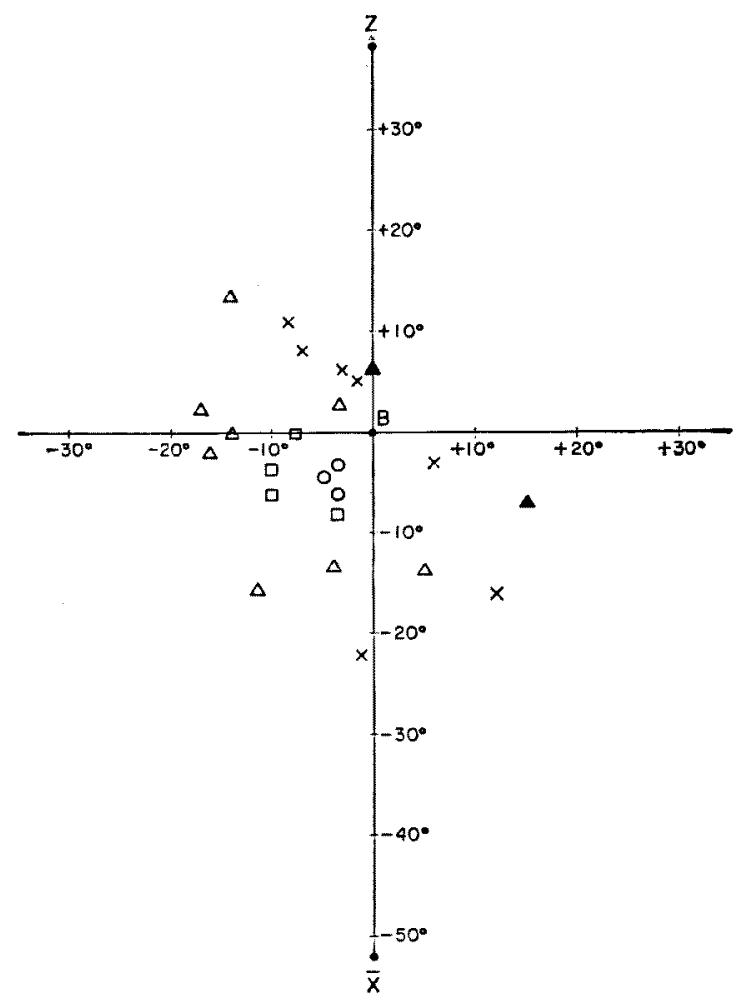

Fig. 4. Dependence of the ellipticity of vibration on orientation near the optic axis B. Notation is the same as Fig. 3.

The region near the optic axis $\mathrm{A}$ is divisible into two parts, one for the right handed vibration, and the other, for the left handed. The two parts are bounded by a line, representing directions for linear vibration, which probably runs from upper-right through $\mathrm{A}$ to lower-left of the figure at a small angle to the trace of the optical plane.

Fig. 4 shows the results of similar measurements made in the directions near the optic axis B. From this figure, it may be seen that, for the light passing in direction near the optic axis B, the ellipticity tends to be smaller than that for the light near A. Here, 
the boundary line between the two parts near B runs probably from upper-left to lower-right.

\section{Interference figure}

The interference figure observed with sections normal to the optic axis $\mathrm{A}$ of babingtonite is peculiar in certain respects. With a section nearly normal to the optic axis $\mathrm{A}$, at the parallel position, a usual black isogyre is seen, crossing the field of view parallel to the vibration direction of polarizer or analyzer. However, instead of isochromatic rings, as ordinarily seen with transparent minerals, there appear rings consisting of various colours; thus, a certain part of one ring is deep green, while the remaining part is faintly coloured, as is illustrated in Fig. 5 (a), Plate I. At the diagonal position, the isogyre changes from black to red, while the deep green part shows no change in colour, as is illustrated in Fig 5 (b), Plate I.

With a monochromatic red light, the above-said deep green portion of the ring is replaced by a dark arc, and the remaining portion is seen only weakly lighted, as shown in Fig. 5 (c), Plate I. With gradual change of colour of light toward blue, the dark arc extends along the ring, and finally, with blue light, dark part forms one continuous ring (Fig, 5 (e), Plate I). At the diagonal position, with red light, the isogyre is obscure (Fig. 5 (d), Plate I). With change of colour as above, the isogyre becomes more and more distinct (Fig. 5 (f), Plate I).

The interference figure for sections nearly normal to the optic axis B is not so peculiar as that for sections normal to A. The isogyre is less clearly seen than in the ordinary case.

Although a thorough explanation of the above peculiar features of the interference figure is not at hand, this may be due to elliptic vibration of light and its strong absorption variation near the optic axis. 


\section{Absorption and pleochroism}

Pleochroism was observed with grains, orientated practically perpendicular to each of the bisectrices $X, Y$ and $Z$, with the following result. Z....pale brown, Y....lilac, X....deep green.

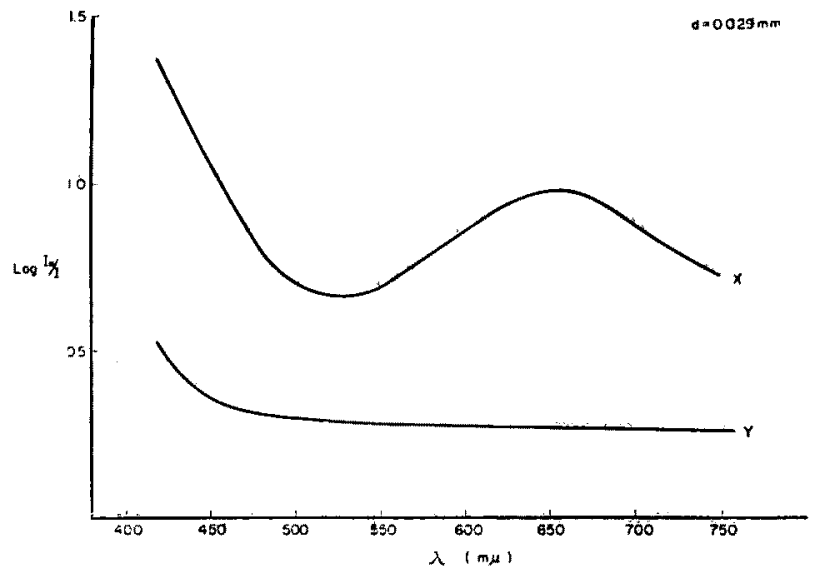

Fig. 6. Variation of the optical density with wavelength for the section practically normal to $Z$.

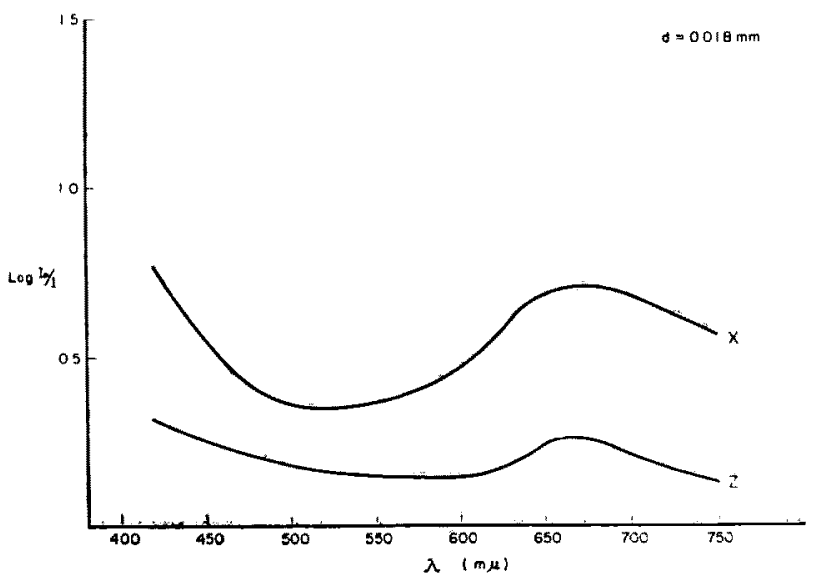

Fig. 7. Variation of the optical density with wavelength for the section practically normal to $\mathrm{Y}$. 
Mineralogical Journal, Vol. 3.

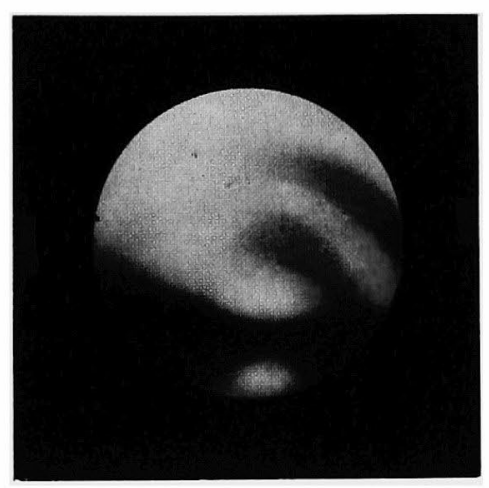

(a)

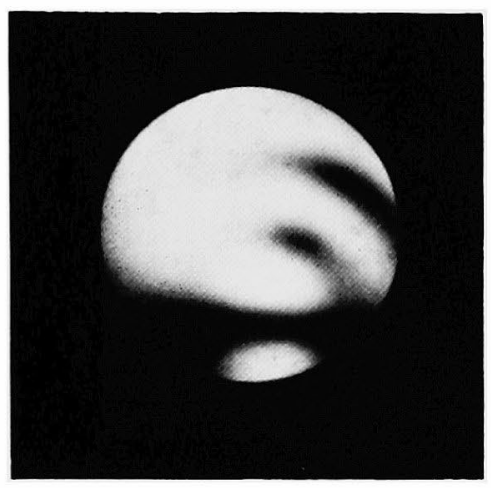

(c)

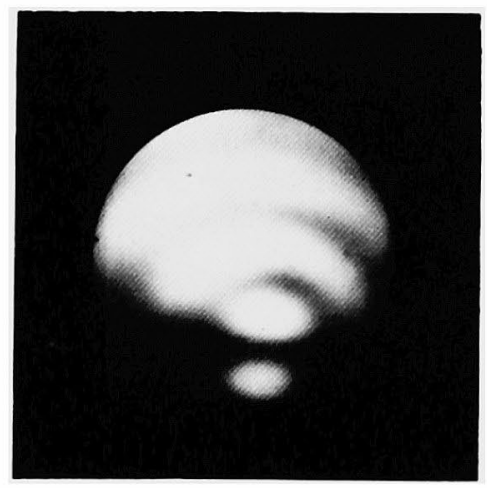

(e)
T. SHODA, Plate I

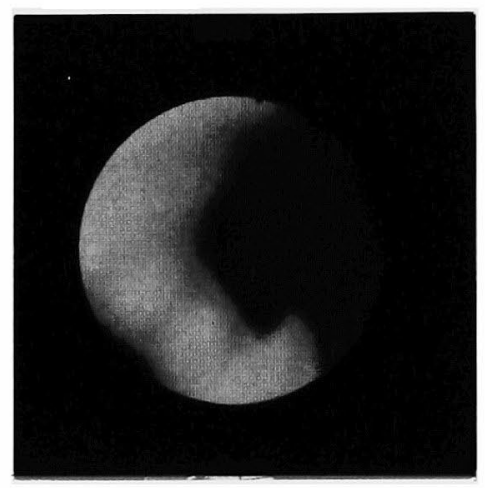

(b)

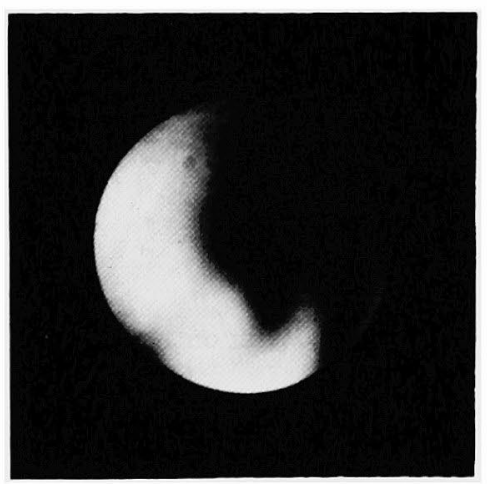

(d)

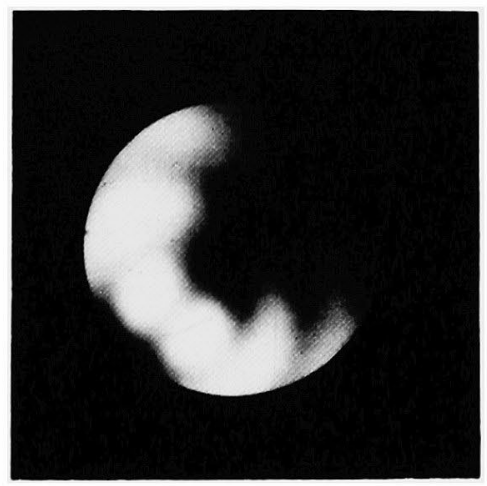

(f)

Fig. 5. Interference figure for a section nearly normal to the optic axis A of Yakuki babingtonite. (a), (b) with white light. (c), (d) with red light. (e), (f) with blue light. 
The variation of optical density with wavelength for these grains was photometrically determined, kindly for the writer, by J. Tanaka. The results are shown in Figs. 6 and 7. It is seen from these figures that the curve for the vibration parallel to $\mathrm{X}$ has a distinct maximum at the position corresponding to the wavelength of about $600 \mathrm{~m} \mu$. The curve for the vibration parallel to $Z$ also has a maximum at the same position as for $\mathrm{X}$, though less remarkable, while the curve for $\mathrm{Y}$ has no appreciable maximum within the range of visible light.

\section{Acknowledgements}

The writer wishes to express his thanks to Emeritus Prof. S. Tsuboi for reading the manuscript with criticism. He also thanks Prof. R. Sadanaga, of this Institute, and Profs. N. Katayama and S. Iwao, College of General Education who made facilities available for this study. He is also indebted to Mr. M. Nishiwaki, the head of the Yakuki mine, who offered the valuable specimen of babingtonite. Special thanks are due to Dr. J. Tanaka of the Institute for Solid State Physics, University of Tokyo for the optical experiments on light absorption. For preparation of sections, the writer is indebted to $\mathrm{Mr}$. T. Watanabe of the Earthquake Research Institute.

\section{REFERENCES}

1) Dauber, A.: Pogg. Ann., 94, 402 (1855).

2) Peacock, M. A.: Amer. Miner., 22, 588 (1937).

3) Richmond, W.E.: Amer. Miner., 22, 630 (1937).

4) Shoda, T.: Miner. Jour., 2, 114 (1957).

5) Shoda, T.: Miner. Jour., 2, 269 (1958).

6) Watanabe, M.: Amer. Jour. Sc., 5th Ser., 4, 159 (1922).

Manuscript received July 15, 1961. 